\title{
Convex-like inequality, homogeneity, subadditivity, and a characterization of $L^{p}$-norm
}

\author{
by Janusz Matkowski and Marek PyCiA (Bielsko-Biała)
}

\begin{abstract}
Let $a$ and $b$ be fixed real numbers such that $0<\min \{a, b\}<1<a+b$. We prove that every function $f:(0, \infty) \rightarrow \mathbb{R}$ satisfying $f(a s+b t) \leq a f(s)+b f(t)$, $s, t>0$, and such that $\limsup _{t \rightarrow 0+} f(t) \leq 0$ must be of the form $f(t)=f(1) t, t>0$. This improves an earlier result in [5] where, in particular, $f$ is assumed to be nonnegative. Some generalizations for functions defined on cones in linear spaces are given. We apply these results to give a new characterization of the $L^{p}$-norm.
\end{abstract}

Introduction. We deal with the functional inequality

$$
f(a s+b t) \leq a f(s)+b f(t),
$$

where $a, b \in \mathbb{R}$ are fixed real numbers such that

$$
0<\min \{a, b\}<1<a+b
$$

and $f$ is a real function defined on $\mathbb{R}_{+}:=[0, \infty)$ or $(0, \infty)$. Our Theorem 2 says that if $f(0)=0, f$ is bounded above in a neighbourhood of 0 , and satisfies this inequality for all $s, t \geq 0$, then $f$ must be a linear function. This improves a result of [6] where $f$ is assumed to be nonnegative. Theorem 1 , the main result of the first section, reads as follows: If $f:(0, \infty) \rightarrow \mathbb{R}$ satisfies the above inequality for all $s, t>0$, and $\lim \sup _{t \rightarrow 0+} f(t) \leq 0$, then $f(t)=f(1) t, t>0$.

In Section 2, using Theorems 1 and 2, we obtain their counterparts for functions defined on convex cones of a linear space. Namely, under some weak regularity conditions an analogue of the above inequality characterizes the Banach functionals.

1991 Mathematics Subject Classification: Primary 26D15, 26A51, 39B72; Secondary $46 \mathrm{E} 30$.

Key words and phrases: functional inequality, subadditive functions, homogeneous functions, Banach functionals, convex functions, linear space, cones, measure space, integrable step functions, $L^{p}$-norm, Minkowski's inequality.

Research supported by the State Committee for Scientific Research (Poland), Grant No. 2 P301 05303. 
Applying these results we give a new characterization of the $L^{p}$-norm (cf. Theorem 3).

1. Functions satisfying a convex-like inequality on $(0, \infty)$ and $\mathbb{R}_{+}$. The main theorem of this section is a refinement of a relevant result of [6] and reads as follows:

THEOREM 1. Let $a, b \in \mathbb{R}$ be fixed and such that condition (1) holds. If $f:(0, \infty) \rightarrow \mathbb{R}$ satisfies

$$
f(a s+b t) \leq a f(s)+b f(t), \quad s, t>0,
$$

and

$$
\limsup _{t \rightarrow 0+} f(t) \leq 0
$$

then $f(t)=f(1) t, t>0$.

Proof. There is no loss of generality in assuming that $a=\min \{a, b\}<1$. Moreover, by (2),

$$
f\left(a s+b(a+b)^{n} t\right) \leq a f(s)+b(a+b)^{n} f(t), \quad s, t>0, n \in \mathbb{N} .
$$

Consequently, we may also assume $b>1$. Now we prove the following

Claim. Under the conditions of Theorem 1 and $a<1<b$ there exists an $M>0$ such that

$$
k a^{n} b^{m} f(t)+M \delta \geq f\left(k a^{n} b^{m} t+\delta\right),
$$

for all $t, \delta>0 ; n, m \in \mathbb{N}, n+m>0 ; k=0, \ldots,\left(\begin{array}{c}n+m \\ m\end{array}\right)$.

To show it, take $c>\max \left\{a+b, a^{-1}\right\}$. By (3) there exists a $t_{0}>0$ such that $f$ is bounded above on the interval $I:=\left(t_{0}, c t_{0}\right)$. Thus, for some $M>0$,

$$
f(t) \leq M t, \quad t \in I .
$$

From $(2), f\left((a+b)^{n} t\right) \leq(a+b)^{n} f(t)$ for all $n \in \mathbb{N}$ and $t>0$. Hence

$$
f(t) \leq M t, \quad t \in \bigcup_{n=0}^{\infty}(a+b)^{n} I .
$$

(For $I \subset \mathbb{R}$ and $\lambda \in \mathbb{R}$ we denote by $\lambda I$ the set $\{\lambda x: x \in I\}$.) Since $c>a+b$, the intervals $(a+b)^{n} I$ and $(a+b)^{n+1} I$ have a nonempty intersection, and, consequently, $\bigcup_{n=0}^{\infty}(a+b)^{n} I=\left(t_{0}, \infty\right)$. This proves that $f(t) \leq M t$ for all $t \in\left(t_{0}, \infty\right)$.

Assume that for some $n \in \mathbb{N}$,

$$
f(t) \leq M t, \quad t \in a^{n} I,
$$


and take $s \in a^{n+1} I$. There exists an increasing sequence $\left(t_{k}\right)$ such that $t_{k} \in a^{n} I(k \in \mathbb{N})$, and $a t_{k} \rightarrow s$. From (2) we have

$$
\begin{aligned}
f(s) & =f\left(a t_{k}+b b^{-1}\left(s-a t_{k}\right)\right) \leq a f\left(t_{k}\right)+b f\left(b^{-1}\left(s-a t_{k}\right)\right) \\
& \leq \text { Mat }_{k}+b f\left(b^{-1}\left(s-a t_{k}\right)\right) .
\end{aligned}
$$

According to (3),

$$
f(s) \leq M a\left(\lim _{k \rightarrow \infty} t_{k}\right)=M s, \quad s \in a^{n+1} I .
$$

Hence, by induction,

$$
f(s) \leq M s, \quad s \in \bigcup_{n=0}^{\infty} a^{n} I .
$$

Since the inequality $c>a^{-1}$ implies that $\bigcup_{n=0}^{\infty} a^{n} I=\left(0, c t_{0}\right)$, it follows that $f(t) \leq M t, t \in\left(0, c t_{0}\right)$. Thus we have proved

$$
f(t) \leq M t, \quad t>0 .
$$

We now show (4) by induction on $N:=n+m$. For $N=1$, (4) follows immediately from (2) and (6), for $k=0$ it reduces to (6). Take $N>1$, $k>0$, choose $k_{1}, k_{2}$ such that

$$
k_{1}+k_{2}=k, \quad k_{1} \leq\left(\begin{array}{c}
n+m-1 \\
m
\end{array}\right), \quad k_{2} \leq\left(\begin{array}{c}
n+m-1 \\
m-1
\end{array}\right),
$$

and suppose that

$$
\begin{aligned}
& k_{1} a^{n-1} b^{m} f(t)+(2 a)^{-1} \delta \geq f\left(k_{1} a^{n-1} b^{m} t+(2 a)^{-1} \delta\right), \\
& k_{2} a^{n} b^{m-1} f(t)+(2 a)^{-1} \delta \geq f\left(k_{2} a^{n} b^{m-1} t+(2 a)^{-1} \delta\right) .
\end{aligned}
$$

Hence, in view of $(2)$, we get

$$
\begin{aligned}
k a^{n} b^{m} f(s) & +M \delta \\
= & a\left(k_{1} a^{n-1} b^{m} f(s)+M(2 a)^{-1} \delta\right)+b\left(k_{2} a^{n} b^{m-1} f(s)+M(2 a)^{-1} \delta\right) \\
\geq & a f\left(k_{1} a^{n-1} b^{m} s+(2 a)^{-1} \delta\right)+b f\left(k_{2} a^{n} b^{m-1} s+(2 a)^{-1} \delta\right) \\
\geq & f\left(a k_{1} a^{n-1} b^{m} s+2^{-1} \delta+b k_{2} a^{n} b^{m-1} s+2^{-1} \delta\right)=f\left(k a^{n} b^{m} s+\delta\right),
\end{aligned}
$$

and the induction completes the proof of our claim.

Now note that the set

$$
\mathbb{D}:=\left\{k a^{n} b^{m}: m, n \in \mathbb{N}, m+n>0, k=0, \ldots,\left(\begin{array}{c}
n+m \\
m
\end{array}\right)\right\}
$$

is dense in $(0, \infty)$. Indeed, if $\log b / \log a$ is irrational, then, in view of Kronecker's Theorem, its subset $\left\{a^{n+1} b^{m}: m, n \in \mathbb{N}\right\}$ is dense in $(0, \infty)$. In the other case there exist $n, m \in \mathbb{N}$ such that $\log b / \log a=-n / m$, which means 
that $a^{n} b^{m}=1$. Since for every $k, j \in \mathbb{N}$,

$$
k a^{j} b=k a^{k n+j} b^{k m+1} \in \mathbb{D},
$$

the set $\mathbb{D}$ contains a dense subset $\left\{k a^{j} b: k, j \in \mathbb{N}\right\}$.

By the definition of $\mathbb{D}$ we can write (4) in the following equivalent form:

$$
\lambda f(t)+M \delta \geq f(\lambda t+\delta), \quad \lambda \in \mathbb{D}, t, \delta>0 .
$$

Now, fix $s, t>0$ and take a sequence $\left(\lambda_{n}\right)$ such that $\lambda_{n} \in \mathbb{D}, \lambda_{n}<s(n \in \mathbb{N})$, $\lim _{n \rightarrow \infty} \lambda_{n}=s$. From (7) we have

$$
\lambda_{n} f(t)+M\left(s-\lambda_{n}\right) t \geq f\left(\lambda_{n} t+\left(s-\lambda_{n}\right) t\right)=f(s t), \quad n \in \mathbb{N} .
$$

Letting $n \rightarrow \infty$ we obtain $s f(t) \leq f(s t)$, which obviously implies that $s f(t)=f(s t)$. Hence $f(s)=f(1) s, s>0$, which completes the proof.

Re mark 1 . It is shown in [6] that every nonnegative function $f$ satisfying (2) with a,b such that (1) holds must be linear. Obviously, this result is a consequence of Theorem 1.

Example 1. Take $a, b>0$ such that $a+b>1$, and $c>0$. Then every function $f:(0, \infty) \rightarrow \mathbb{R}$ such that $c \leq f(t) \leq c(a+b), t>0$, satisfies (2). This shows that the condition (3) in Theorem 1 is essential.

Note that (3) can be considerably weakened if (2) is assumed to hold for all nonnegative $s$ and $t$. Namely, we have the following

Theorem 2. Let $a, b \in \mathbb{R}$ satisfy (1). If $f: \mathbb{R}_{+} \rightarrow \mathbb{R}$ satisfies

$$
f(a s+b t) \leq a f(s)+b f(t), \quad s, t \geq 0,
$$

and

(i) $f(0)=0$;

(ii) $f$ is bounded above in a right vicinity of 0 ,

then $f(t)=f(1) t, t>0$.

This result is an immediate consequence of Theorem 1 and the following

Lemma 1. Let $a, b \in \mathbb{R}$ satisfy (1). Suppose that $f: \mathbb{R}_{+} \rightarrow \mathbb{R}$ satisfies

$$
f(a s+b t) \leq a f(s)+b f(t), \quad s, t \geq 0 .
$$

Then

(i) $f(0) \geq 0$.

(ii) If, moreover, $f(0)=0$ and $f$ is bounded above in a right vicinity of 0 , then condition (3) holds.

Pro of. (i) is obvious. To prove (ii) suppose that, say, $a=\min \{a, b\}$ and observe that, by the boundedness above of $f$ to the right of 0 , we have

$$
c:=\limsup _{t \rightarrow 0+} f(t)<\infty .
$$


Setting in the assumed inequality $s=0$ and making use of the condition $f(0)=0$, we get $f(a t) \leq a f(t)$ for all $t \geq 0$. It follows that $c \leq a c$. Since $a<1$ we hence get $c \leq 0$, which was to be shown.

EXAmple 2. The function $f: \mathbb{R}_{+} \rightarrow \mathbb{R}$ given by $f(t)=t^{-1}, t>0$, and $f(0)=0$ satisfies (2) for all $a, b \in \mathbb{R}$ such that condition (1) holds. This shows that, in Theorem 2, the assumption of $f$ being bounded above in a (right) neighbourhood of 0 is indispensable.

EXAmPLE 3. Let $a, b>0$ be rational. Then every discontinuous additive function $f: \mathbb{R} \rightarrow \mathbb{R}$ satisfies (2). It is well known that the graph of $f$ is a dense subset of the plane (cf. for instance Aczél-Dhombres [1], p. 14). This also shows that the regularity assumptions in Theorems 1 and 2 are necessary.

2. Some generalizations for functions defined on cones. In this section, using Theorems 1 and 2, we prove their more general counterparts.

Let $\boldsymbol{X}$ be a real linear space. A set $\boldsymbol{C} \subset \boldsymbol{X}$ is said to be a convex cone in $\boldsymbol{X}$ iff $\boldsymbol{C}+\boldsymbol{C} \subset \boldsymbol{C}$ and $t \boldsymbol{C} \subset \boldsymbol{C}$ for all $t>0$.

A functional $\boldsymbol{p}: \boldsymbol{C} \rightarrow \mathbb{R}$ is called subadditive iff

$$
\boldsymbol{p}(\boldsymbol{x}+\boldsymbol{y}) \leq \boldsymbol{p}(\boldsymbol{x})+\boldsymbol{p}(\boldsymbol{y}), \quad \boldsymbol{x}, \boldsymbol{y} \in \boldsymbol{C},
$$

and positively homogeneous iff

$$
\boldsymbol{p}(t \boldsymbol{x})=t \boldsymbol{p}(\boldsymbol{x}), \quad t>0, \boldsymbol{x} \in \boldsymbol{C} .
$$

In the sequel the functionals satisfying both these conditions (the so-called Banach functionals) will appear frequently.

Denote by $\boldsymbol{o}$ the zero vector of $\boldsymbol{X}$. If $\boldsymbol{C}$ is a convex cone in $\boldsymbol{X}$ and $\boldsymbol{o} \in \boldsymbol{C}$, then $t \boldsymbol{C} \subset \boldsymbol{C}$ for all $t \geq 0$.

Corollary 1. Let $\boldsymbol{X}$ be a real linear space and $\boldsymbol{C} \subset \boldsymbol{X}$ a convex cone such that $\boldsymbol{o} \in \boldsymbol{C}$. Suppose that $a, b \in \mathbb{R}$ are fixed and $0<\min \{a, b\}<1<$ $a+b$. Then a function $\boldsymbol{p}: \boldsymbol{C} \rightarrow \mathbb{R}$ is subadditive and positively homogeneous if and only if

(i) $\boldsymbol{p}(\boldsymbol{o})=0$;

(ii) for every $\boldsymbol{x} \in \boldsymbol{C}$, the function $(0, \infty) \ni t \rightarrow \boldsymbol{p}(t \boldsymbol{x})$ is bounded above in a right vicinity of 0 ; and

$$
\boldsymbol{p}(a \boldsymbol{x}+b \boldsymbol{y}) \leq a \boldsymbol{p}(\boldsymbol{x})+b \boldsymbol{p}(\boldsymbol{y}), \quad \boldsymbol{x}, \boldsymbol{y} \in \boldsymbol{C} .
$$

Proof. First suppose that $\boldsymbol{p}$ satisfies (i), (ii), and (8). Then for every fixed $\boldsymbol{x} \in \boldsymbol{C}$ the function $f: \mathbb{R}_{+} \rightarrow \mathbb{R}$ defined by $f(t):=\boldsymbol{p}(t \boldsymbol{x}), t \geq 0$, satisfies all the assumptions of Theorem 2. Consequently, $\boldsymbol{p}(t \boldsymbol{x})=f(t)=$ $f(1) t=t \boldsymbol{p}(\boldsymbol{x})$ for all $t \geq 0$, which means that $\boldsymbol{p}$ is positively homogeneous. 
Now the subadditivity of $\boldsymbol{p}$ is a consequence of (8). Since the converse is obvious, the proof is complete.

In a similar way, applying Theorem 1 , we get

Corollary 2. Let $\boldsymbol{X}$ be a real linear space and $\boldsymbol{C} \subset \boldsymbol{X}$ a convex cone. Suppose that $a, b \in \mathbb{R}$ are fixed and $0<\min \{a, b\}<1<a+b$. Then $a$ function $\boldsymbol{p}: \boldsymbol{C} \rightarrow \mathbb{R}$ is subadditive and positively homogeneous if and only if it satisfies (8) and

$$
\limsup _{t \rightarrow 0+} \boldsymbol{p}(t \boldsymbol{x}) \leq 0, \quad \boldsymbol{x} \in \boldsymbol{C} .
$$

Let $\boldsymbol{X}$ be a real linear space, $\boldsymbol{C} \subset \boldsymbol{X}$ a convex cone in $\boldsymbol{X}$ and $\phi: \boldsymbol{C} \rightarrow \mathbb{R}$. We say that $\phi$ is a linear functional on $\boldsymbol{C}$ iff $\phi(\boldsymbol{x}+\boldsymbol{y})=\phi(\boldsymbol{x})+\phi(\boldsymbol{y})$ for all $\boldsymbol{x}, \boldsymbol{y} \in \boldsymbol{C}$, and $\phi(t \boldsymbol{x})=t \phi(\boldsymbol{x})$ for all $t>0, \boldsymbol{x} \in \boldsymbol{C}$. Note that if $\phi \not \equiv 0$, then $\phi^{-1}(\{1\})=\{\boldsymbol{x} \in \boldsymbol{C}: \phi(\boldsymbol{x})=1\}$ is a nonempty convex subset of $\boldsymbol{C}$, and put $\operatorname{supp}(\phi):=\{\boldsymbol{x} \in \boldsymbol{C}: \phi(\boldsymbol{x}) \neq 0\}$.

The term "linear functional" is legitimate in view of the following

Remark 2. Let $\phi: C \rightarrow \mathbb{R}$ be additive and positively homogeneous on a cone $\boldsymbol{C} \subset \boldsymbol{X}$ such that $\boldsymbol{C} \cap(-\boldsymbol{C})=\{\boldsymbol{o}\}$. Denote by $\boldsymbol{Y}$ the linear span of $\boldsymbol{C}$. It is easy to check that there exists a unique linear functional $\Phi: \boldsymbol{Y} \rightarrow \mathbb{R}$ such that $\left.\Phi\right|_{C}=\phi$.

Proposition. Let $\boldsymbol{X}$ be a real linear space, $\boldsymbol{C} \subset \boldsymbol{X}$ a cone in $\boldsymbol{X}$ such that $\boldsymbol{C} \cap(-\boldsymbol{C})=\{\boldsymbol{o}\}$, and $\phi: \boldsymbol{C} \rightarrow \mathbb{R}$ a linear functional on $\boldsymbol{C}$ such that $\phi \geq 0$ on $\boldsymbol{C}$. Suppose that $a, b \in \mathbb{R}$ are fixed and $0<\min \{a, b\}<1<a+b$. If $H: \operatorname{supp}(\phi) \rightarrow \mathbb{R}$ satisfies

$$
H(a \boldsymbol{x}+b \boldsymbol{y}) \leq a H(\boldsymbol{x})+b H(\boldsymbol{y}), \quad \boldsymbol{x}, \boldsymbol{y} \in \operatorname{supp}(\phi),
$$

and

$$
\limsup _{t \rightarrow 0+} H(t \boldsymbol{x}) \leq 0, \quad \boldsymbol{x} \in \operatorname{supp}(\phi),
$$

then $H$ is positively homogeneous and subadditive.

Moreover, the function $h: \phi^{-1}(1) \rightarrow \mathbb{R}$ defined by

$$
h(\boldsymbol{x}):=H(\boldsymbol{x}), \quad \boldsymbol{x} \in \phi^{-1}(1),
$$

is convex,

$$
H(\boldsymbol{x})=\phi(\boldsymbol{x}) h(\boldsymbol{x} / \phi(\boldsymbol{x})), \quad \boldsymbol{x} \in \operatorname{supp}(\phi),
$$

and

(9) $\phi(\boldsymbol{x}+\boldsymbol{y}) h\left(\frac{\boldsymbol{x}+\boldsymbol{y}}{\phi(\boldsymbol{x}+\boldsymbol{y})}\right)$

$$
\leq \phi(\boldsymbol{x}) h\left(\frac{\boldsymbol{x}}{\phi(\boldsymbol{x})}\right)+\phi(\boldsymbol{y}) h\left(\frac{\boldsymbol{y}}{\phi(\boldsymbol{y})}\right), \quad \boldsymbol{x}, \boldsymbol{y} \in \operatorname{supp}(\phi) .
$$


P r o o f. It is easy to check that $\operatorname{supp}(\phi)$ is a convex cone in $\boldsymbol{X}$. Therefore the first conclusion is a consequence of Corollary 2 .

To prove the remaining assertion note that $z \in \phi^{-1}(1)$ if and only if there is an $\boldsymbol{x} \in \operatorname{supp}(\Phi)$ such that $\boldsymbol{z}=\boldsymbol{x} / \phi(\boldsymbol{x})$. Take any $\boldsymbol{x} \in \operatorname{supp}(\phi)$. By the positive homogeneity of $H$ and the definition of $h$ we have

$$
H(\boldsymbol{x})=\phi(\boldsymbol{x}) H(\boldsymbol{x} / \phi(\boldsymbol{x}))=\phi(\boldsymbol{x}) h(\boldsymbol{x} / \phi(\boldsymbol{x})) .
$$

Hence, the subadditivity of $H$ gives (9). This inequality implies the convexity of $h$, and the proof is complete.

Remark 3. Taking in the Proposition $\boldsymbol{X}=\mathbb{R}^{k}, \boldsymbol{C}=\mathbb{R}_{+}^{k}, k \in \mathbb{N}$, and the functional $\phi: \boldsymbol{C} \rightarrow \mathbb{R}_{+}, \phi(\boldsymbol{x})=\phi\left(x_{1}, \ldots, x_{k}\right)=x_{i}$, the projection on the $x_{i}$-axis, $i \in\{1, \ldots, k\}$, we get the result proved in [5] (cf. also [6]). Moreover, it is shown in [5] that inequality (9) with $\phi$ being the projection characterizes the convex functions $h$ defined on $(0, \infty)^{k-1}$ and generalizes Minkowski's and Hölder's inequalities. Thus inequality (9) may also be interpreted as a generalization of these two fundamental inequalities.

3. An application to a characterization of the $L^{p}$-norm. For a measure space $(\Omega, \Sigma, \mu)$ denote by $\boldsymbol{S}=\boldsymbol{S}(\Omega, \Sigma, \mu)$ the linear space of all $\mu$-integrable step functions $\boldsymbol{x}: \Omega \rightarrow \mathbb{R}$ and by $\boldsymbol{S}_{+}=\boldsymbol{S}_{+}(\Omega, \Sigma, \mu)$ the set of all nonnegative $\boldsymbol{x} \in \boldsymbol{S}$. If $\varphi, \psi: \mathbb{R}_{+} \rightarrow \mathbb{R}_{+}$are one-to-one, onto and $\varphi(0)=0$ then the functional $\mathbf{P}_{\varphi, \psi}: S \rightarrow \mathbb{R}$ given by the formula

$$
\mathbf{P}_{\varphi, \psi}(\boldsymbol{x}):=\psi\left(\int_{\Omega} \varphi \circ|\boldsymbol{x}| d \mu\right), \quad \boldsymbol{x} \in \boldsymbol{S},
$$

is well defined. The goal of this section is to prove the following

THEOREM 3. Let $(\Omega, \Sigma, \mu)$ be a measure space with at least two disjoint sets of finite and positive measure. Suppose that $a, b \in \mathbb{R}$ are fixed numbers such that

$$
0<\min \{a, b\}<1<a+b,
$$

and $\varphi, \psi: \mathbb{R}_{+} \rightarrow \mathbb{R}_{+}$are one-to-one, onto, continuous at 0 and $\varphi(0)=$ $\psi(0)=0$. If

$$
\mathbf{P}_{\varphi, \psi}(a \boldsymbol{x}+b \boldsymbol{y}) \leq a \mathbf{P}_{\varphi, \psi}(\boldsymbol{x})+b \mathbf{P}_{\varphi, \psi}(\boldsymbol{y}), \quad \boldsymbol{x}, \boldsymbol{y} \in \boldsymbol{S}_{+},
$$

then $\varphi(t)=\varphi(1) t^{p}$ and $\psi(t)=\psi(1) t^{1 / p}(t \geq 0)$ for some $p \geq 1$.

Proof. Take any $\boldsymbol{x} \in \boldsymbol{S}_{+}$. Then there exist $n$ pairwise disjoint sets $A_{1}, \ldots, A_{n} \in \Sigma$ of finite measure, and $x_{1}, \ldots, x_{n} \in \mathbb{R}_{+}$such that $\boldsymbol{x}=$ $\sum_{k=1}^{n} x_{k} \chi_{A_{k}}$. ( $\chi_{A}$ stands for the characteristic function of the set $A$.) From the definition of $\mathbf{P}_{\varphi, \psi}$ we have

$$
\mathbf{P}_{\varphi, \psi}(t \boldsymbol{x})=\psi\left(\int_{\Omega} \varphi \circ|t \boldsymbol{x}| d \mu\right)=\psi\left(\sum_{k=1}^{n} \varphi\left(t x_{k}\right) \mu\left(A_{k}\right)\right), \quad t>0 .
$$


The continuity of $\varphi$ and $\psi$ at zero and $\varphi(0)=\psi(0)=0$ imply that $\lim _{t \rightarrow 0+} \mathbf{P}_{\varphi, \psi}(t \boldsymbol{x})=0$. By Corollary 2 the functional $\mathbf{P}_{\varphi, \psi}$ is positively homogeneous, i.e.

$$
\mathbf{P}_{\varphi, \psi}(t \boldsymbol{x})=t \mathbf{P}_{\varphi, \psi}(\boldsymbol{x}), \quad \boldsymbol{x} \in \boldsymbol{S}_{+}, t>0,
$$

and subadditive:

$$
\mathbf{P}_{\varphi, \psi}(\boldsymbol{x}+\boldsymbol{y}) \leq \mathbf{P}_{\varphi, \psi}(\boldsymbol{x})+\mathbf{P}_{\varphi, \psi}(\boldsymbol{y}), \quad \boldsymbol{x}, \boldsymbol{y} \in \boldsymbol{S}_{+} .
$$

By our assumption on the measure space, there are two disjoint sets $A, B \in$ $\Sigma$ of finite positive measure. Put $\alpha:=\mu(A)$ and $\beta:=\mu(B)$. Taking $\boldsymbol{x}:=$ $x_{1} \chi_{A}+x_{2} \chi_{B}$ with $x_{1}, x_{2} \geq 0$ in (10), we get

$$
\psi\left(\alpha \varphi\left(t x_{1}\right)+\beta \varphi\left(t x_{2}\right)\right)=t \psi\left(\alpha \varphi\left(x_{1}\right)+\beta \varphi\left(x_{2}\right)\right) .
$$

Since $\psi$ and $\varphi$ are bijective we can write this equation in the following equivalent form:

$$
\begin{aligned}
\alpha \varphi\left(t \varphi^{-1}\left(x_{1}\right)\right)+\beta \varphi\left(t \varphi^{-1}\left(x_{2}\right)\right) & \\
& =\psi^{-1}\left(t \psi\left(\alpha x_{1}+\beta x_{2}\right)\right), \quad t>0, x_{1}, x_{2} \geq 0 .
\end{aligned}
$$

Substituting here first $x_{2}=0$, and next $x_{1}=0$ we get

$$
\begin{array}{ll}
\alpha \varphi\left(t \varphi^{-1}\left(x_{1}\right)\right)=\psi^{-1}\left(t \psi\left(\alpha x_{1}\right)\right), & t>0, x_{1} \geq 0, \\
\beta \varphi\left(t \varphi^{-1}\left(x_{2}\right)\right)=\psi^{-1}\left(t \psi\left(\beta x_{2}\right)\right), & t>0, x_{2} \geq 0 .
\end{array}
$$

The relations (13) and (14) allow us to write (12) in the form $\psi^{-1}\left(t \psi\left(\alpha x_{1}\right)\right)+\psi^{-1}\left(t \psi\left(\beta x_{2}\right)\right)=\psi^{-1}\left(t \psi\left(\alpha x_{1}+\beta x_{2}\right)\right), \quad t>0, x_{1}, x_{2} \geq 0$, or, equivalently,

$$
\psi^{-1}\left(t \psi\left(x_{1}\right)\right)+\psi^{-1}\left(t \psi\left(x_{2}\right)\right)=\psi^{-1}\left(t \psi\left(x_{1}+x_{2}\right)\right), \quad t>0, x_{1}, x_{2} \geq 0 .
$$

Thus, for every $t>0$, the function $\psi^{-1} \circ(t \psi)$ is additive. Since it is nonnegative, it follows that for every $t>0$ there is an $m(t)>0$ such that

$$
\psi^{-1}(t \psi(u))=m(t) u, \quad u>0 .
$$

Writing an analogous equation for every $s>0$ we have

$$
\psi^{-1}(s \psi(u))=m(s) u, \quad u>0 .
$$

Composing separately the functions on the left- and the right-hand sides of these equations we obtain

$$
\psi^{-1}(s t \psi(u))=m(s) m(t) u, \quad u>0 .
$$

Replacing $t$ by st in (15) we get

$$
\psi^{-1}(s t \psi(u))=m(s t) u, \quad u>0 .
$$

The last two equations imply that $m(s t)=m(s) m(t), s, t>0$, i.e. $m$ : $(0, \infty) \rightarrow(0, \infty)$ is a solution of the multiplicative Cauchy equation. Putting 
$u=1$ in (15) we get $m(t)=\psi^{-1}(t \psi(1)), t>0$. It follows that $m$ is a bijection of $(0, \infty)$, and, of course, the inverse function to $m$,

$$
m^{-1}(t)=\psi(t) / \psi(1), \quad t>0,
$$

is multiplicative. The continuity of $\psi$ at 0 implies that there exists a $p \in \mathbb{R}$, $p \neq 0$, such that $m^{-1}(t)=t^{1 / p}$ for all $t>0$. Hence

$$
\psi(t)=\psi(1) t^{1 / p}, \quad t>0 .
$$

Inserting this into (13) we have $\alpha \varphi\left(t \varphi^{-1}\left(x_{1}\right)\right)=\alpha x_{1} t^{p}$ for all $t>0$ and $x_{1} \geq 0$. Taking $x_{1}:=\varphi^{-1}(1)$ we obtain

$$
\varphi(t)=\varphi(1) t^{p}, \quad t>0 .
$$

Now, for the above power functions $\varphi$ and $\psi,(11)$ reduces to the classical Minkowski inequality. It follows that $p \geq 1$. This completes the proof.

Remark 4. To prove that (13) and (14) imply that $\varphi$ and $\psi$ are the inverse power functions we could apply some results proved in [4].

A similar result holds if $\mathbf{P}_{\varphi, \psi}$ satisfies the opposite inequality to that of Theorem 3. One should emphasize that, in this case, the regularity assumptions on functions $\varphi$ and $\psi$ are superfluous. Namely, we have

THEOREM 4. Let $(\Omega, \Sigma, \mu)$ be a measure space with at least two disjoint sets of finite positive measure. Suppose that $a, b \in \mathbb{R}$ are fixed with $0<$ $\min \{a, b\}<1<a+b$, and $\varphi, \psi: \mathbb{R}_{+} \rightarrow \mathbb{R}_{+}$are one-to-one, onto, and $\varphi(0)=0$. If

$$
\mathbf{P}_{\varphi, \psi}(a \boldsymbol{x}+b \boldsymbol{y}) \geq a \mathbf{P}_{\varphi, \psi}(\boldsymbol{x})+b \mathbf{P}_{\varphi, \psi}(\boldsymbol{y}), \quad \boldsymbol{x}, \boldsymbol{y} \in \boldsymbol{S}_{+},
$$

then $\varphi(t)=\varphi(1) t^{p}$ and $\psi(t)=\psi(1) t^{1 / p}(t \geq 0)$ for some $p, 0<p \leq 1$.

Proof. Since $-\mathbf{P}_{\varphi, \psi}$ satisfies the opposite inequality to (16) and $\left(-\mathbf{P}_{\varphi, \psi}\right)(\boldsymbol{x}) \leq 0$ for all $\boldsymbol{x} \in \boldsymbol{S}_{+}$, Corollary 2 implies that $\mathbf{P}_{\varphi, \psi}$ is positively homogeneous, and superadditive on $\boldsymbol{S}_{+}$, i.e.

$$
\mathbf{P}_{\varphi, \psi}(\boldsymbol{x}+\boldsymbol{y}) \geq \mathbf{P}_{\varphi, \psi}(\boldsymbol{x})+\mathbf{P}_{\varphi, \psi}(\boldsymbol{y}), \quad \boldsymbol{x}, \boldsymbol{y} \in \boldsymbol{S}_{+} .
$$

Arguing in the same way as in the proof of Theorem 3 we show that the function $m:(0, \infty) \rightarrow(0, \infty), m(t)=\psi^{-1}[t \psi(1)], t>0$, is multiplicative on $(0, \infty)$.

As in the proof of Theorem 3, take disjoint sets $A, B \in \Sigma$ of finite positive measure, and put $\alpha:=\mu(A)$ and $\beta:=\mu(B)$. Substituting, in (17), $\boldsymbol{x}, \boldsymbol{y} \in \boldsymbol{S}_{+}$ such that

$$
\boldsymbol{x}:=x_{1} \chi_{A}+x_{2} \chi_{B}, \quad \boldsymbol{y}:=y_{1} \chi_{A}+y_{2} \chi_{B}, \quad x_{1}, x_{2}, y_{1}, y_{2} \geq 0,
$$

we get

$\psi\left(\alpha \varphi\left(x_{1}+y_{1}\right)+\beta \varphi\left(x_{2}+y_{2}\right)\right) \geq \psi\left(\alpha \varphi\left(x_{1}\right)+\beta \varphi\left(x_{2}\right)\right)+\psi\left(\alpha \varphi\left(y_{1}\right)+\beta \varphi\left(y_{2}\right)\right)$ 
for all $x_{1}, x_{2}, y_{1}, y_{2} \geq 0$. Take arbitrary $s, t \geq 0$. Putting

$$
x_{1}=\varphi(s / \alpha)^{-1}, \quad x_{2}=y_{1}=0, \quad y_{2}=\varphi(t / \beta)^{-1},
$$

and making use of the assumption that $\varphi(0)=0$, we get

$$
\psi(s+t) \geq \psi(s)+\psi(t), \quad s, t \geq 0 .
$$

Hence $\psi$ is increasing, and, consequently, a homeomorphism of $\mathbb{R}_{+}$. It follows that the multiplicative function $m$ is a homeomorphism of $(0, \infty)$.

Now, by an argument as in the proof of Theorem 3, we show that there exists a $p \in \mathbb{R}, p \neq 0$, such that $\psi(t)=\psi(1) t^{1 / p}$ and $\varphi(t)=\varphi(1) t^{p}, t>0$. Substituting these functions into (16) we obtain the "companion" of the Minkowski inequality which is known to hold only for $p \in(0,1]$. This concludes the proof.

Remark 5. Theorems 3 and 4 can be interpreted to be converses of the Minkowski inequalities (cf. [7] and [8] where converses of Minkowski's inequality other than Theorem 3 are given).

\section{References}

[1] J. Aczél and J. Dhombres, Functional Equations in Several Variables, Encyclopedia Math. Appl. 31, Cambridge University Press, Cambridge, Sydney, 1989.

[2] E. Hille and R. S. Phillips, Functional Analysis and Semi-groups, Amer. Math. Soc. Colloq. Publ. 31, Amer. Math. Soc., Providence, R.I., 1957.

[3] M. Kuczma, An Introduction to the Theory of Functional Equations and Inequalities. Cauchy's equation and Jensen's inequality, Prace Nauk. Uniw. Śl. 489, Polish Scientific Publishers, 1985.

[4] J. Matkowski, On a characterization of $L^{p}$-norm, Ann. Polon. Math. 50 (1989), 137-144.

[5] -, A functional inequality characterizing convex functions, conjugacy and a generalization of Hölder's and Minkowski's inequalities, Aequationes Math. 40 (1990), $168-180$.

[6] -, Functional inequality characterizing nonnegative concave functions in $(0, \infty)$, ibid. 43 (1992), 219-224.

[7] —, The converse of the Minkowski's inequality theorem and its generalization, Proc. Amer. Math. Soc. 109 (1990), 663-675.

[8] -,$L^{p}$-like paranorms, in: Selected Topics in Functional Equations and Iteration Theory, Proc. Austrian-Polish Seminar, Graz, 1991, Grazer Math. Ber. 316 (1992), 103-135.

DEPARTMENT OF MATHEMATICS

RAFOWA 21 TECHNICAL UNIVERSITY

43-300 BIELSKO-BIAłA, POLAND

WILLOWA 2

43-309 BIELSKO-BIAłA, POLAND 